\title{
Outcomes of Radial Artery Grafts Without Postoperative Calcium Channel Blocker Administration
}

\author{
Zachary DeBoard ${ }^{1}$, Hyung Chan Kim² , and James K. Brevig ${ }^{1}$ \\ ${ }^{1}$ Providence Regional Medical Center Everett \\ ${ }^{2}$ Washington State University Elson S Floyd College of Medicine
}

December 22, 2021

\begin{abstract}
Background: Guidelines encourage oral pharmacologic antispasmodic therapy for patients receiving a radial artery conduit during coronary artery bypass grafting. We review our experience with radial artery conduits without the postoperative use of calcium channel blocker therapy. Methods: A single-center, retrospective review patients undergoing isolated coronary artery bypass grafting with at least one radial artery conduit over a three-year period was performed. Patient demographic, operative, and post-discharge data were collected. Development of angina or angina equivalent symptoms, imaging suggestive of radial conduit failure, or percutaneous intervention to the territory grafted by a radial artery was considered to represent graft failure. Patients were evaluated for primary outcomes through 90 days postoperatively and followed for 1 year overall. Results: 264 adult patients underwent first-time, isolated coronary artery bypass grafting with use of a radial artery conduit. Three patients were observed to have radial graft occlusions during the first 90 days, all of which were attributed to technical issues. No patients required addition of a calcium channel blocker \& no additional patients underwent imaging or intervention for radial graft failure during 1 year of follow up. Conclusions: Avoidance of postoperative calcium channel blocker therapy in patients receiving a radial artery graft was not associated with a high incidence of imaging-confirmed or clinically suggested conduit failure.
\end{abstract}

Title: Outcomes of Radial Artery Grafts Without Postoperative Calcium Channel Blocker Administration Running Title: Radial Artery Calcium Channel

Zach M. DeBoard, MD ${ }^{1,2}$, Brian HC Kim, BS ${ }^{2}$, James K. Brevig, $\mathrm{MD}^{1,2}$

1. Cardiac \& Thoracic Surgery, Providence Regional Medical Center Everett, Everett, WA

2. Washington State University Elson S. Floyd College of Medicine, Pullman, WA

Word Count: 2870

Corresponding Author:

Zach M. DeBoard, MD

Cardiac \& Thoracic Surgery

1330 Rockefeller Avenue

Suite 400

Everett, WA 98201

Phone: 425-261-4950 
Fax: 425-261-4951

Email: zachary.deboard@providence.org

Sources of Funding \& Conflicts of Interest: none

Background: Guidelines encourage oral pharmacologic antispasmodic therapy for patients receiving a radial artery conduit during coronary artery bypass grafting. We review our experience with radial artery conduits without the postoperative use of calcium channel blocker therapy.

Methods: A single-center, retrospective review patients undergoing isolated coronary artery bypass grafting with at least one radial artery conduit over a three-year period was performed. Patient demographic, operative, and post-discharge data were collected. Development of angina or angina equivalent symptoms, imaging suggestive of radial conduit failure, or percutaneous intervention to the territory grafted by a radial artery was considered to represent graft failure. Patients were evaluated for primary outcomes through 90 days postoperatively and followed for 1 year overall.

Results: 264 adult patients underwent first-time, isolated coronary artery bypass grafting with use of a radial artery conduit. Three patients were observed to have radial graft occlusions during the first 90 days, all of which were attributed to technical issues. No patients required addition of a calcium channel blocker \& no additional patients underwent imaging or intervention for radial graft failure during 1 year of follow up.

Conclusions: Avoidance of postoperative calcium channel blocker therapy in patients receiving a radial artery graft was not associated with a high incidence of imaging-confirmed or clinically suggested conduit failure.

Introduction:

Common approaches to surgical coronary artery revascularization include using a single arterial conduit (left internal mammary artery) in addition to the saphenous vein. Multi-arterial grafting has been shown to improve survival, graft patency, and reduce the need for repeat revascularization. ${ }^{1-4}$ Current guidelines for coronary revascularization encourage a multiple arterial conduit approach. ${ }^{5}$

One option for an additional arterial conduit is the radial artery (RA). Despite the reported benefits of RA conduits there remains modest adoption of their use. ${ }^{6}$ One area of concern with RA conduits is postoperative spasm that can lead to decreased patency. Preventative maneuvers to limit spasm intraoperatively include topical application of antispasmodics and vasodilators. ${ }^{7}$ There remains debate over the utility of postoperative oral anti-spasmodic medication administration for RA conduits. Recommendations exist for oral calcium channel blocking (CCB) agents ranging between 1-3 months postoperatively in patients receiving a RA conduit. ${ }^{5}$ Nevertheless, additional medications may lend to hypotension, side effects, or the complications inherent to polypharmacy. ${ }^{5,8}$ While reports have suggested there may be a benefit to patency with postoperative CCB use in patients receiving a RA conduit, the data is based on a modest cohort of patients. We present our experience with RA conduits without postoperative CCB use.

Methods:

The Providence/St. Joseph's Health System Institutional Review Board deemed this study exempt. Using the Providence Regional Medical Center Everett's electronic medical record (EMR) and our data submitted to the Society of Thoracic Surgeons all adult (age $>18$ years) patients undergoing isolated CABG and receiving at least one RA conduit between January 1, 2017 and January 1, 2020 were reviewed. Patients were excluded if they underwent emergency operations, re-operative sternotomy for CABG, concomitant aortic or valvular procedures, or lacked adequate follow up of at least 90 days. Ninety days was selected for the primary evaluation period as most reports of included in the STS recommendations limit provision of CCBs to this time frame. ${ }^{5}$

Patient demographics and operative/hospital data including number of radial arteries used, coronary arteries bypassed, length of stay, and mortality were reviewed. Discharge medications and outpatient clinic notes were reviewed to evaluate for the addition of a CCB or nitrate as well as new cardiac imaging (angiography, 
echocardiogram, stress test) for purposes of angina or equivalent symptoms. Any diagnostic imaging study results pertaining to evaluation of graft patency (angiography, echocardiogram) were also reviewed. Our EMR facilitated chart review as it integrates numerous regional hospital and outpatient clinical throughout our region as well as partner institutions within the Providence/St. Joseph's Health System.

Primary outcomes of radial conduit failure over the 90 day follow-up were determined angiographically if a patient required percutaneous intervention to either the RA conduit or native coronary distribution grafted with a RA conduit. Patients with echocardiography or stress testing revealing ischemia in the territory grafted with a RA conduit were also considered to have RA graft failure/dysfunction. Initiation of a CCB or nitrate for angina or new angina equivalent symptoms (dyspnea, fatigue) was used as a surrogate for angiographically determined graft failure. Imaging evaluation and percutaneous intervention were evaluated for 1 year from the date of surgery.

Radial arteries were harvested as a pedicled conduit via an open technique with ultrasonic shears and/or hemoclips to ligate radial vessel branches. The artery is further prepared by proximal cannulation and is then flushed with a solution of $15 \mathrm{mg}$ verapamil, $6.8 \mathrm{mg}$ nitroglycerin, $8 \mathrm{mEq}$ sodium bicarbonate, and 1600 units heparin per liter of lactated ringers. The radial artery is then bathed in the same solution until it is brought to the field for grafting. End-to-side anastomoses to the native coronary artery and ascending aorta are performed using a running 7-0 and 6-0 polypropylene suture for the distal, and proximal anastomoses, respectively. Sequential anastomoses were conducted in a side-to-side fashion with running 7-0 polypropylene. The proximal and distal extents of the parallel veins along the radial artery pedicle are occluded with hemoclips at the completion of the anastomoses. Additional cardioplegia is administered via the conduit upon completion of the distal anastomosis. Trans-sonic flow probe analyses are conducted after separation from cardiopulmonary bypass.

Results:

496 patients underwent first-time CABG at our institution during the study period. Of these, 264 patients had at least one radial artery conduit ( $53.2 \%$ of all first-time CABG performed). Of the 264 patients, 37 had both radial arteries harvested, and 30 had sequential anastomoses performed. A total of 331 distal anastomoses with radial grafts were performed. The majority of patients were male and most operations were performed in the setting of an acute coronary syndrome. Patient demographic data appears in table 1.

The majority of radial arteries were used to bypass left-sided coronary lesions. Obtuse marginal vessels were the most commonly bypassed target $(\mathrm{n}=132 ; 39.9 \%$ of distal anastomoses) followed the right posterior descending artery ( $\mathrm{n}=81 ; 24.5 \%$ of distal anastomoses). Radial graft anastomotic data appears in table 2 .

Three patients with preoperative CCB (diltiazem) use were maintained on the medication postoperatively. Two patients were discharged on a nitrate (isosorbide) for long-term use in the setting of peripheral arterial disease. Over the 90-day follow up window, no patients were initiated on calcium channel blocker or nitrate therapy.

Eighty-three patients (31\%) were discharged on dual antiplatelet therapy (DAPT) of which clopidogrel was the predominant medication in addition to aspirin. Twenty-one of these patients were on preoperative DAPT for recent drug eluting stent placement. The remaining patients were placed on DAPT for poor quality coronary targets. Intra- and post-operative data appear in Table 3.

Seven patients underwent repeat coronary angiography during the follow up window. Of these, three were observed to have issues related to the RA conduit ( $1.13 \%$ of patients, $0.3 \%$ of total distal radial graft anastomoses). One patient within 30 days postoperative had a RA graft occlusion and two other patients were found to have RA graft occlusions within the 31-90 day postoperative period. Four internal mammary artery grafts and three saphenous vein conduits were found to be non-functional in the angiographic evaluations. No further percutaneous intervention or echocardiographic evaluation for concern of dysfunctional grafts or development of angina or equivalent symptoms occurred on any patient through the 90-day to 1-year post-operative time period. Angiographically observed RA findings appear in table 4. 
The cohort's 30-day mortality was $0.75 \%(2 / 264)$ and 1-year mortality was $1.14 \%$ (3/264). The 30-day observed-to-expected mortality ratio was 1.05. Causes of in-hospital death included ischemic bowel due to chronic abdominal visceral arterial disease (postoperative day 27) and acute on chronic respiratory failure (postoperative day 52). The single out of hospital mortality was due to respiratory arrest from accidental overdose of chronic pain medication on postoperative day 56 .

Conclusions:

We present a cohort of patients undergoing CABG with RA conduits over a 3-year period. We utilize RA conduits in over $50 \%$ of CABG compared to $6-7 \%$ at similar programs in the US. ${ }^{6}$ A category IIa, level B recommendation by the STS encourages use of a second arterial conduit for patients undergoing CABG..$^{5}$ Recent literature has demonstrated improved survival, reduced need for repeat revascularization, and fewer major adverse cardiac and cerebrovascular events (MACCE) in patients receiving an additional arterial graft, of which the RA is an option. ${ }^{1-4}$ With regard to the RA, STS guidelines provide a category IIa, level C recommendation for oral pharmacologic manipulation (predominantly a CCB) postoperatively. ${ }^{5}$ Our group does not prescribe CCBs postoperatively unless a patient is taking these medications prior to surgery for pre-existing issues. Many centers however have been documented to routinely provide CCB therapy. ${ }^{9}$ The addition of a CCB may result in patients failing to be up-titrated on necessary medications such as beta blockers or angiotensin converting enzyme inhibitors as well as being subject to side effects such as hypotension, headaches, or flushing.

A recent review of randomized control trials comparing CCB use to no CCB administration evaluated 732 patients with RA grafts. Angiographic follow up was available for 443 patients and demonstrated statistically improved angiographic patency of RA grafts at 36 months for patients receiving a CCB (99.1\% vs. $78.6 \%) .{ }^{10}$ Nevertheless, two of the studies cited in the review failed to demonstrate improved RA graft patency in patients receiving $\mathrm{CCB}$ therapy immediately post $\mathrm{CABG}$, including those provided CCB therapy for 1 year or more. ${ }^{11,12}$ These findings are further supported by the lack of association with development of an angiographic RA "string sign" in patients taking CCB or not. ${ }^{13}$

As routine angiographic evaluation of all patients during a postoperative time frame is not feasible in our institution we used addition of a CCB or nitrate as well as angina/angina-equivalent symptoms and imaging (echocardiography, scintigraphy) suggestive of ongoing radial-graft territory ischemia as surrogates for angiographically-determined graft failure. Patients who underwent angiographic evaluation of or percutaneous coronary intervention to the coronary artery bypassed with a radial conduit were also reviewed. During this time, our cohort's patency rate for radial grafts was $98.8 \%$ of patients. Previous pooled reports of RA patency rates note between $95-100 \%$ patency prior to six months and between 87-99\% at 6-36 months. ${ }^{14}$ We consider the failure of our cohort's RA conduits to be technical in nature as the lesions were observed at anastomotic sites and occurred early in the postoperative period.

We believe overall RA patency may be more so influenced by technical and anatomic factors rather than administration of CCBs postoperatively. We use an open, pedicled harvest technique employing ultrasonic shears and striving for minimal conduit manipulation. Gaudino and colleagues reviewed methods of RA harvest and observed mixed results as to pedicled versus skeletonized techniques with regards to patency. ${ }^{15}$ We also aim use RAs to graft larger targets and coronaries with significant proximal stenosis $(>80-90 \%)$ or those with a chronic total occlusion. Lower patency of RA grafts to right-sided targets, coronary arteries with modest lesions, or coronary arteries with poor runoff territory and heavy disease burden demonstrates the effects of competitive flow and target selection as contributing factors to poor RA conduit patency. ${ }^{15-17}$ In a review by He and Taggart the authors note that evolution in surgical techniques may be more contributory to RA graft patency than systemic CCB use. ${ }^{7}$

Our group does not routinely prescribe dual antiplatelet therapy (DAPT) after CABG (31\% prescribed DAPT). The majority of DAPT administration in the current study postoperatively was from a single surgeon. Our group prescribes postoperative DAPT for patients taking such medications preoperatively or for heavily diseased coronary targets and modest flow vein grafts. The effect of DAPT on RA patency 
remains unknown.

Our study has a variety of limitations. First, the data was retrospectively collected and does not include a comparison group. Second, we are unable to obtain universal angiographic evaluation of all CABG patients with radial conduits at a specific postoperative interval. Finally, patients may have had angiographic coronary evaluation or intervention post surgery at another institution not captured by our EMR review.

In summary our cohort of patients undergoing CABG with RA conduits without postoperative CCB administration demonstrated expected graft patency without increased need for intervention or clinical indications of RA conduit failure over the study period.

References:

1. Hosono M, Murakami T, Hirai H, Sasaki Y, Suehiro S, Shibata T. The Risk Factor Analysis for Late Graft Failure of Radial Artery Graft in Coronary Artery Bypass Grafting. Ann Thorac Cardiovas. 2019;25(1):3238.

2. Deb S, Cohen EA, Singh SK, Une D, Laupacis A, Fremes SE. Radial Artery and Saphenous Vein Patency More than 5 Years After Coronary Artery Bypass Surgery: Results from RAPS (Radial Artery Patency Study). J Am Coll Cardiol. 2012;60(1):28-35.

3. Gaudino M, Benedetto U, Fremes S, et. al, RADIAL Investigators. Radial-Artery of Saphenous-Vein Grafts in Coronary-Artery Bypass Surgery. N Engl J Med. 2018;378:2069-2077.

4. Rocha RV, Tam DY, Karkhanis R, et al. Multiple Arterial Grafting Is Associated With Better Outcomes for Coronary Artery Bypass Grafting Patients. Circulation. 2018;138(19):2081-2090.

5. Aldea GS, Bakaeen FG, Pal J, et al. The Society of Thoracic Surgeons Clinical Practice Guidelines on Arterial Conduits for Coronary Artery Bypass Grafting. Ann Thorac Surg. 2016;101:801-809.

6. Schwann TA, Habib RH, Wallace A, et al. Operative Outcomes of Multiple-Arterial Versus Single-Arterial Coronary Bypass Grafting. Ann Thorac Surg. 2018;105(4):1109-1119.

7. He G-W, Taggart DP. Spasm In Arterial Grafts in Coronary Artery Bypass Grafting Surgery. Ann Thorac Surg. 2016;101(3):1222-1229.

8. Gupta P, Patel P, Strauch B, et al. Risk Factors for Nonadherence to Antihypertensive Treatment. Hypertension. 2017;69(6):1113-1120.

9. Myers MF, Fremes SE. Prevention of Radial Artery Graft Spasm: A Survey of Canadian Surgical Centres. Can J Cardiol. 2003;19(6):677-681.

10. Gaudino M, Benedetto U, Fremes SE, et al. Effect of Calcium-Channel Blocker Therapy on Radial Artery Grafts After Coronary Bypass Surgery. J Am Coll Cardiol. 2019;73(18):2299-2306.

11. Gaudino M, Glieca F, Luciani N, Alessandrini F, Possati G. Clinical and Angiographic Effects of Chronic Calcium Channel Blocker Therapy Continued Beyond First Postoperative Year in Patients With Radial Artery Grafts. Circulation. 2001;104(12 Suppl 1):I64-67.

12. Gaudino M, Luciani N, Nasso G, Salica A, Canosa C, Possati G. Is Postoperative Calcium Channel Blocker Therapy Needed in Patients With Radial Artery Grafts? J Thorac Cardiovasc Surg. 2005;129(3):532535.

13. Miwa S, Desai N, Koyama T, Chan E, Cohen EA, Fremes SE, Radial Artery Patency Study Investigators. Radial Artery Angiographic String Sign: Clinical Consequences and the Role of Pharmacologic Therapy. Ann Thorac Surg. 2006;81(1):112-118.

14. Verma S, Szmitko PE, Weisel RD, et al. Should Radial Arteries Be Used Routinely For Coronary Artery Bypass Grafting? Circulation. 2004;110(5):40-46. 
15. Gaudino M, Crea F, Cammertoni F, Mazza A, Toesca A, Massetti M. Technical Issues in The Use of The Radial Artery As A Coronary Artery Bypass Conduit. Ann Thorac Surg. 2014;98:2247-2254.

16. Maniar HS, Sundt TM, Barner HB, et al. Effect of Target Stenosis And Location on Radial Artery Graft Patency. J Thorac Cardiovasc Surg. 2002;123(1):45-52.

17. Gaudino M, Fremes S, Schwann TA, Tatoulis J, Wingo M, Tranbaugh RF. Technical Aspects of The Use of The Radial Artery in Coronary Artery Bypass Surgery. Ann Thorac Surg. 2019;108(2): 613-622.

Table 1. Patient Demographics $(\mathrm{n}=264)$

\begin{tabular}{lll}
\hline Demographic & $\mathrm{n}=(\%)$ or median= (range) & $\mathrm{n}=(\%)$ or median= (range) \\
\hline Age & $65(29-83)$ & \\
Male & $223(84.5)$ & \\
Urgent operation (for acute coronary syndrome) & $148(56.1)$ & $148(56.1)$ \\
Preoperative ejection fraction (\%) & $55(15-70)$ & $55(15-70)$ \\
Preoperative creatinine & $0.96(0.47-8.46)$ & $0.96(0.47-8.46)$ \\
Preoperative hematocrit & $41(24.9-55.8)$ & $41(24.9-55.8)$ \\
Preoperative hemoglobin A1c & $6.1(4.6-15.0)$ & $6.1(4.6-15.0)$ \\
Body mass index & $29.69(17.74-56.94)$ & $29.69(17.74-56.94)$ \\
Diabetes mellitus & $127(48.1)$ & $127(48.1)$ \\
Insulin-dependent & $40(15.2)$ & $40(15.2)$ \\
Obstructive sleep apnea & $67(25.4)$ & $67(25.4)$ \\
Chronic obstructive pulmonary disease & $42(15.9)$ & $42(15.9)$ \\
Mild & $31(11.7)$ & $31(11.7)$ \\
Moderate / Severe & $11(4.2)$ & $11(4.2)$ \\
STS predicted risk of mortality (\%) & $0.71(0.19-9.78)$ & $0.71(0.19-9.78)$ \\
\hline
\end{tabular}

Table 2. Radial conduit data $(\mathrm{n}=264$ patients; 331 total distal anastomoses $)$

\begin{tabular}{lll}
\hline Location of distal anastomosis & $\mathrm{n}=(\%$ of total radial anastomoses) & $\mathrm{n}=(\%$ of total radial anastomoses $)$ \\
\hline Right coronary artery & $30(9.0)$ & \\
Right posterior descending artery & $81(24.4)$ & \\
Posterior lateral branch & $18(5.4)$ & $18(5.4)$ \\
Obtuse marginal (not further defined) & $65(19.6)$ & $65(19.6)$ \\
Obtuse marginal 1 & $33(9.9)$ & $33(9.9)$ \\
Obtuse marginal 2 & $32(9.6)$ & $32(9.6)$ \\
Obtuse marginal 3 & $2(0.6)$ & $2(0.6)$ \\
Diagonal & $33(9.9)$ & $33(9.9)$ \\
Ramus intermedius & $27(8.1)$ & $27(8.1)$ \\
Left anterior descending & $12(3.6)$ & $12(3.6)$ \\
\hline
\end{tabular}

Table 3. Intra \& postoperative data $(\mathrm{n}=264$ patients)

\begin{tabular}{lll}
\hline Demographic & $\mathrm{n}=(\%)$ or median $=($ range $)$ & $\mathrm{n}=(\%)$ or median= (range) \\
\hline Total radial distal anastomoses & 331 & \\
Sequential anastomoses & $30(11.4)$ & $37(14.0)$ \\
Bilateral radial artery used & $37(14.0)$ & $105(43-294)$ \\
Cardiopulmonary bypass time (minutes) & $105(43-294)$ &
\end{tabular}




\begin{tabular}{lll}
\hline Demographic & $\mathrm{n}=(\%)$ or median= (range) & $\mathrm{n}=(\%)$ or median= (range) \\
\hline Cross clamp time (minutes) & $89(36-212)$ & $89(36-212)$ \\
Intra-aortic balloon pump & $11(4.2)$ & $11(4.2)$ \\
Postoperative length of stay of patients discharged (days) & $5(2-33)$ & $5(2-33)$ \\
Dual antiplatelet therapy on discharge & $83(31.4)$ & $83(31.4)$ \\
Preoperative dual antiplatelet therapy & $21(7.9)$ & $21(7.9)$ \\
\hline
\end{tabular}

Table 4. Angiographic Findings in Patients With Radial Artery Occlusion

\begin{tabular}{llll}
\hline Patient \# & Postoperative Day \# & Coronary Angiogram Report & Coronary Angiogram Rep \\
\hline 1 & 66 & Radial occluded at proximal anastomosis & \\
2 & 40 & Radial "atretic" with distal anastomotic subtotal occlusion & \\
3 & 13 & Radial with subtotal occlusion at proximal anastomosis & Radial with subtotal occl \\
\hline
\end{tabular}

